http://jmscr.igmpublication.org/home/ ISSN (e)-2347-176x ISSN (p) 2455-0450

crossref DOI: https://dx.doi.org/10.18535/jmscr/v8i2.10

\title{
Anatomical and positional evaluation of patellofemoral joint and its association with proximal patellar tendinitis on MRI
}

Authors

\author{
Dr Satyapraveen Samantula ${ }^{1}$, Dr G S Kejriwal ${ }^{2}$, Dr Anil Devara ${ }^{3}$, Dr K Anil Kumar ${ }^{4}$ \\ ${ }^{1}$ Post Graduate, Dept of Radiology, Maharajah's Institute of Medical Sciences \\ ${ }^{2}$ Professor, Dept of Radiology, Maharajah's Institute of Medical Sciences \\ ${ }^{3,4}$ Asst Professor, Dept of Radiology, Maharajah's Institute of Medical Sciences
}

\begin{abstract}
Objective: To determine variation in structure and alignment of the knee between patients with proximal patellar tendinitis (PT) and a control group, with emphasis on the patellofemoral joint on MRI.

Materials \& Methods: Case group included retrospective inclusion of 25 patients with diagnosed PT on MRI. Control group included 50 patients with no radiological or clinical evidence of $P T$ and underwent MRI of the Knee. Physically active patients and controls were matched for age and gender.

Two radiologists evaluated Knee MRIs assessing parameters such as patellar \& trochlear morphology, patellofemoral \& tibiofemoral alignment. Student's $t$ test was used to find out the differences in parameters between cases and controls. Assessment of PT and its association with MRI parameters was done using Logistic regression.

Results: Wiberg angle (Subchondral) was higher in cases than controls (139.8 0.5 vs. $134.5 \pm 7.1 ; p=$ 0.003). Insall-Salvati ratio concerning patellar height was different between cases and controls (1.29 \pm 0.23 vs. $1.13 \pm 0.20 ; p=0.002)$, so does Caton-Deschamps index $(1.3 \pm 0.20$ vs. $1.1 \pm 0.30 ; p=0.004)$. Significant association with tendinitis were found [odds ratios (CI 95\%)] for patellar structure [1.1 (1.0, 1.2)] and patellar height [1.3 (1.0, 1.7)] using logistic regression.

Conclusions: Patellar tendinitis is found to be significantly associated with increased subchondral patellar wiberg angle and patellar height.

Keywords: Patellar tendinits; Patellofemoral Joint; MRI; Patella; Sinding Larsoen Johansson disease.
\end{abstract}

\section{Introduction}

Tendon injuries due to overuse are not limited to just competitive athletes but also affects many working population and regular sports participants, the underlying pathology being tendinosis or collagen degeneration ${ }^{1}$. More than half of the overuse injuries in athletes involve tendons, tendon sheaths, and tendon insertions ${ }^{2,3}$. Most common affected sites include patellar and achillies tendon. Injuries at patellar tendon include
Patellar tendinopathy, peritendinitis, tendinosis, patellar apicitis (jumper's knee) and OsgoodSchlatter disease (apophysitis at the tibial tuberosity), most common being Osgood-Schlatter disease and patellar tendinopathy ${ }^{4}$. Evaluation of extensor mechanism of the knee includes quadriceps muscle \& tendon, patella, patellar tendon and supra \& infrapatellar fat pad. Proximal patellar tendinitis also called as Jumpers knee or patellar tendinopathy occurs mainly at the 
proximal insertion of patellar tendon to the distal aspect of patella due to the repetitive straining of the tendon occurring from sudden knee extensions and repeated quadriceps muscle eccentric contractions ${ }^{5,6}$. Studies found that almost half of athletes with jumpers knee have onset of symptoms before the age of 20 years $^{7}$ and a significant correlation between patellar tendinopathy, leg length inequality and patella alta $^{8}$. So, anatomical \& alignment issues are hypothesized to play a role in overuse tendinopathy. This altered morphology of the knee especially at the patellofemoral compartment may affect load distribution to the extensor mechanism which includes patellar tendon'. Magnetic resonance imaging (MRI) enables evaluation of many anatomical and alignment factors for assessment of patellofemoral joint ${ }^{10}$. There is good correlation between histologic changes in the proximal patellatendon of jumper's knee with both MRI \& US ${ }^{11}$.

The biomechanical analysis of tendinitis needs further studies. The etiology of insertional patellar tendinopathy is recently well analyzed, and it remains to be clarified whether tensile strains ${ }^{12}$ or compressive loads and impingement ${ }^{13}$ play a major role in disease process ${ }^{14}$. We intend to find out that differences in radiologically detected patellofemoral factors related to its morphology and alignment in patients with patellar tendinitis and those with normal anatomy.

\section{Material and Methods}

Subjects: This is a retrospectively done cross sectional study. Informed consent requirement was waived off as this is a retrospective study and the same was approved by the institutional review committee. Records of the patients who underwent knee MRI in our institution from 2016 to 2018 were retrieved from the archives of our workstation. These records are searched for the reports containing diagnosis of patellar tendinitis, jumpers' knee or patellar tendinopathy. Age group of subjects included is in between 20 and 50 years.
Cases \& Controls: Cases showed definitive features of proximal patellar tendinitis. Cases have classic clinical history of patellar tendinitis including pain, tenderness \& swelling in the inferior pole of patella and radiological features on MRI. Control group included same age group with no clinical features of patellar tendinitis and has been referred to our department for evaluation of other knee problems MRI. Both groups are physically active.

Exclusion Criteria: Patients with knee osteoarthritis, a history of recent knee injury, knee surgery, exhibiting severe metallic artifacts on MRI, partial findings of patellar tendinitis on MRI, or a complete tear of the patellar tendons quadriceps or were excluded. Identification capability of association between a continuous variable and patellar tendinitis with is with a statistical power of $80 \%$.

MRI Technique: Examination of Knee is done using 1.5T MRI magnet with dedicated knee coil $\&$ the patient in supine position. (Philips Ingenia 1.5T). Routine Knee MRI protocol included axial, sagittal \& coronal planes with intermediate weighted turbo spin-echo sequences with fat suppression (FS) (TR $3968 \mathrm{~ms}$, TE $30 \mathrm{~ms}$, slice thickness $3 \mathrm{~mm}$, matrix $512 \times 512$, FOV $=15$ ), STIR imaging sequence (TR $3655 \mathrm{~ms}$, TI $160 \mathrm{~ms}$, TE $60 \mathrm{~ms}$ ), proton density-weighted turbo spinecho sequence (TR $2000 \mathrm{~ms}$, TE $15 \mathrm{~ms}$, slice thickness $3 \mathrm{~mm}$, matrix $512 \times 512$, FOV 15), a coronal T1W sequence (TR $582 \mathrm{~ms}$, TE $8 \mathrm{~ms}$, slice thickness $3 \mathrm{~mm}$, matrix $512 \times 512$, FOV 16).

MRI Assessment: Two radiologists with expertise in musculoskeletal imaging evaluated patellar tendons in axial, coronal and sagittal FS images. Finings correlating positively for patellar tendinitis include thickening of the proximal patellar tendon near its insertion on patella relative to mid and distal portion, intrasubstance signal changes in FS images especially in deep portion of patellar tendon and central insertion point on patella. Associated findings include bone marrow edema predominantly at the inferior pole of the patella, adjacent Hoffa's fat pad infiltration, and 
partial-thickness tear of the proximal patellar tendon at its deep aspect. Absence of tendon thickening with the presence of intrasubstance signal is not considered as a case in the current study because normal patellar tendon has a wide range of appearances due to intrinsic fiber differences \& technical factors during MRI acquisition $^{15}$.

All cases showed positive $\&$ definitive findings on MRI and controls were perfectly normal. Patellar and trochlear anatomy, patellofemoral alignment were evaluated using 10 MRI based parameters by two radiologists. Anatomical parameters include Wiberg's subchondral angle \& index for patella (Figure 2,8) and trochlear sulcus angle (Figure 3), the medial-lateral trochlear facets length ratio \& lateral inclination angle for Trochlea. Patellofemoral alignment parameters comprise of Caton-Deschamps (Figure 7) \& Insall-Salvati ratios (Figure 5) for patellar height. The tibial tuberosity-trochlear groove (TT-TG) distance, lateral patellar displacement \& patellar tilt. Marginal osteophytes noted at medial and lateral aspects of patella and trochlea were excluded from measurements. In the evaluation of patellar height, enthesiophytes present at proximal patellar tendon insertion as well as osteophytes at proximal and distal aspect of patellar subchondral bone were excluded in the calculations. Influence of patellofemoral alignment by femorotibial alignment is neutralized by assessing femorotibial angle (Figure 6) on the Cor T1W images. This is measured by the angle formed between the diaphysis axis of the proximal tibia \& distal femur at the middle of the femorotibial compartments. Reproducibility of MRI parameters was done by randomly analyzing 20 samples from our study. MRIs were reassessed 2 months after both radiologists have reported to avoid recognition bias.

Statistical Analysis: Intraclass coefficient correlations is applied for assessment of reproducibility of various MRI parameters by radiologists. Students t test is done for evaluation difference in MRI parameters of cases \& controls.
Logistic regression is further applied to find out association between patellar tendinitis and measured parameters. The MRI parameters showing differences between cases and controls with a $\mathrm{p}$ value $<0.1$ in student's $\mathrm{t}$ test were included in logistic regression. For potential confounders, multi adjustments were performed. C-statistics and R2 statistics used for measurement of quality of these adjustments was measured using. Statistical significance was set at 0.05. All analyses were done using SPSS software.

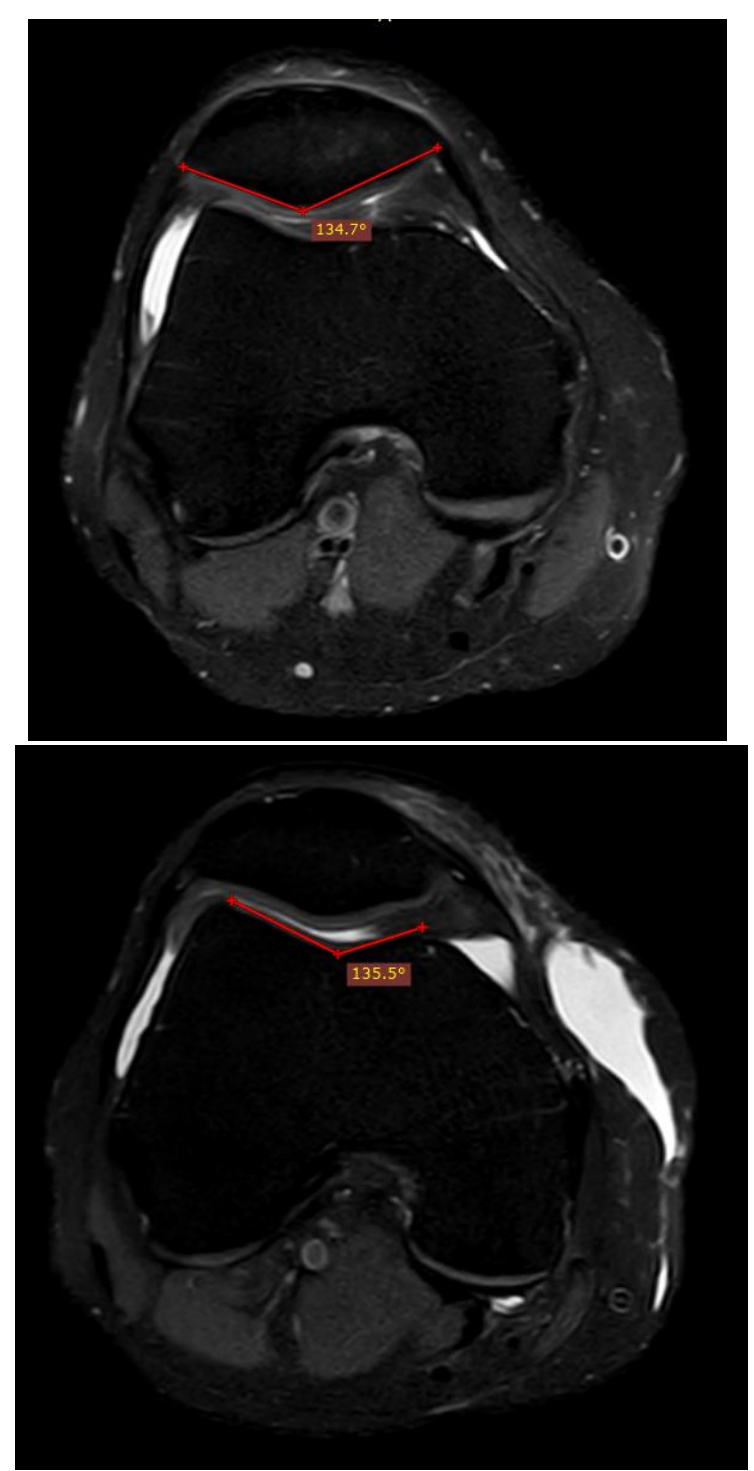

Figure 2, 3: Subchondral Wiberg Angle \& Trochlear Sulcus Angle 


\section{JMSCR Vol||08||Issue||02||Page 39-46||February}
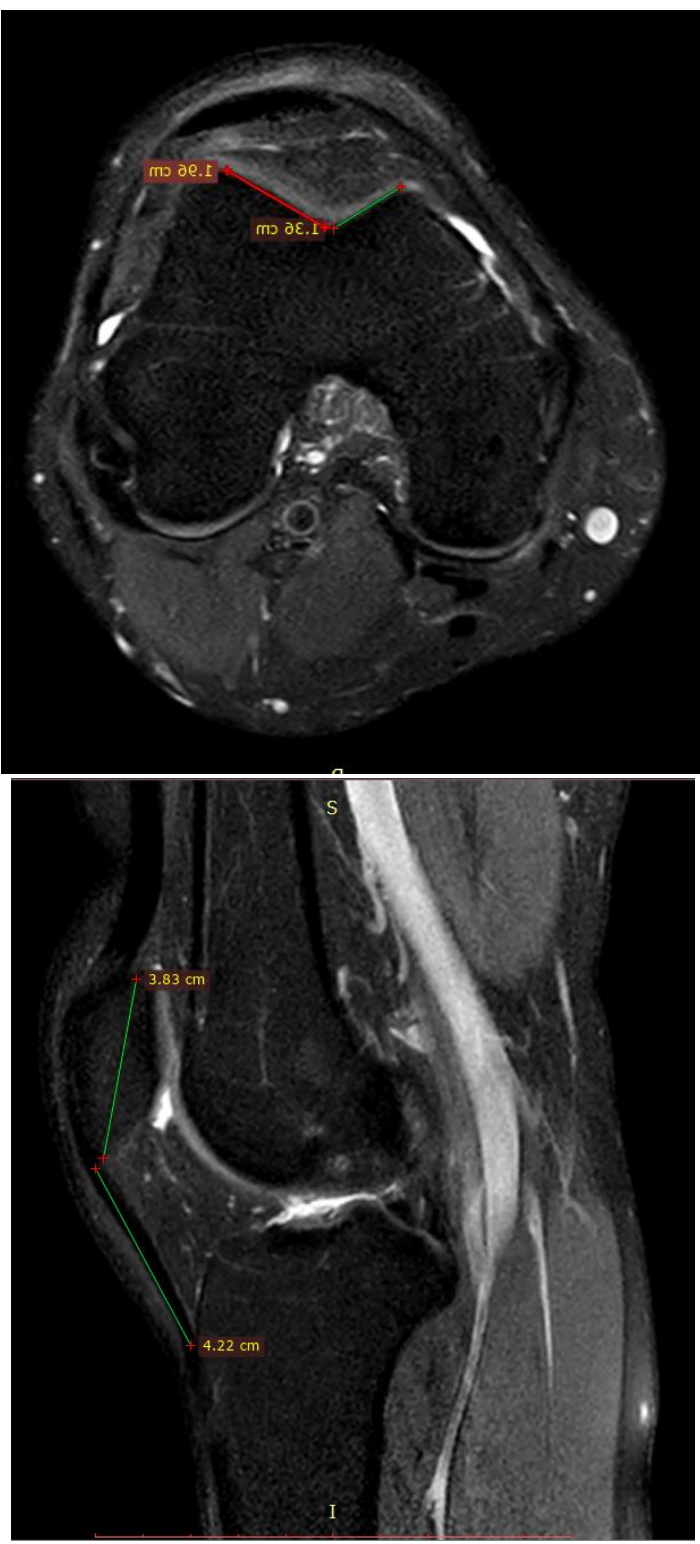

Figure 4,5: Medial/Lateral Trochlear Length Ratio \&Insall-Salvati ratio

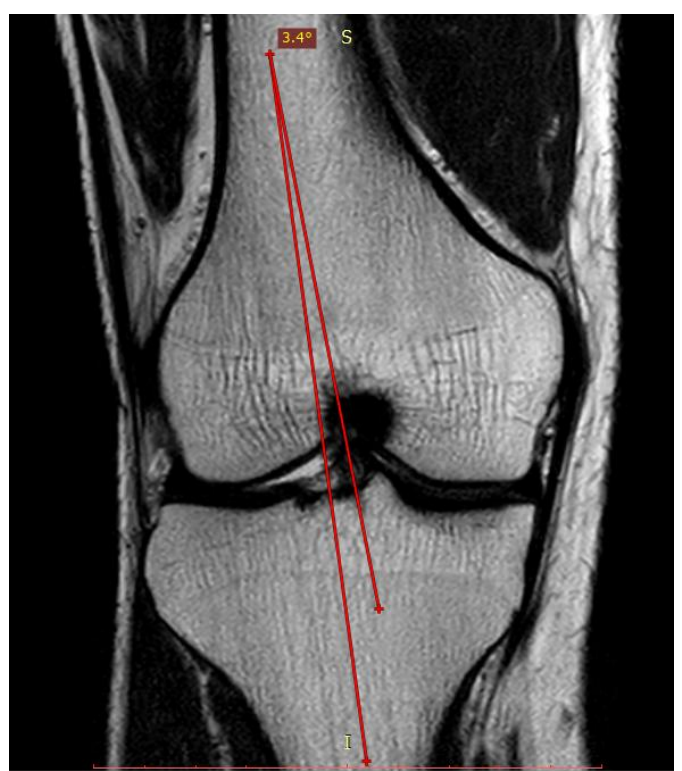

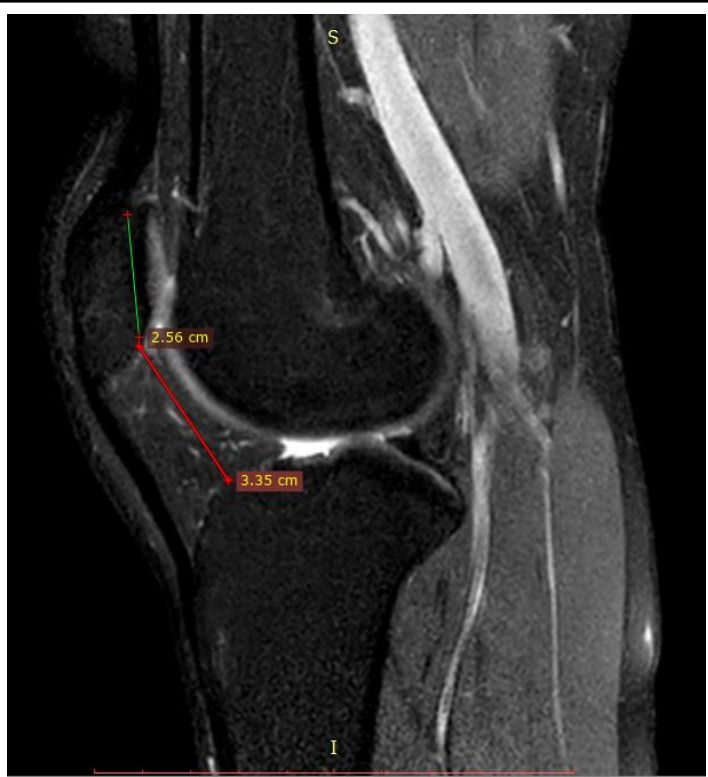

Figure 6,7: Femoro Tibial Angle\&CatonDeschamps ratio

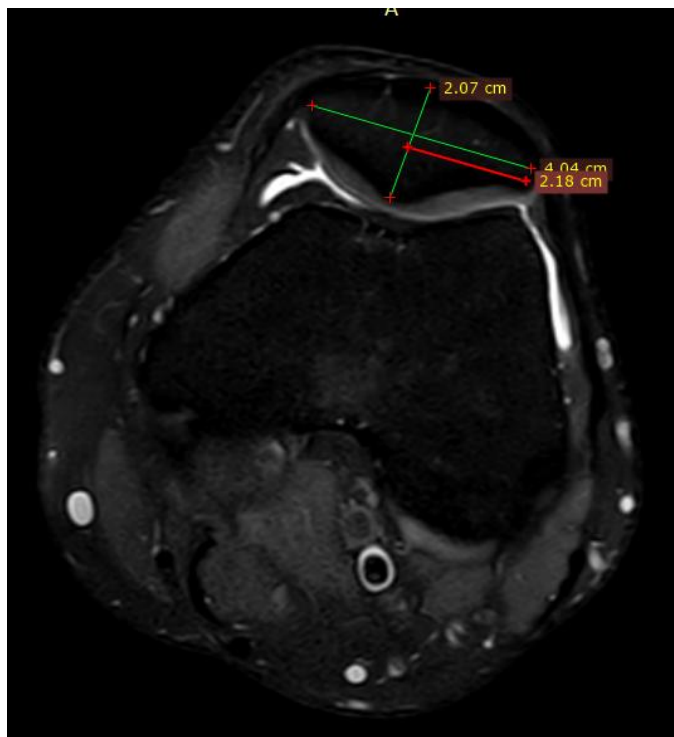

Figure 8: Subchondal - Wiberg Index

\section{Results}

Table 1 demonstrates differences in MRI parameters of Patellofemoral anatomy and alignment between cases and controls done using students $t$ test. No significant differences between the groups with respect to age (Mean 35.2 years \pm 9.1 standard deviation (SD) for cases vs. mean 35.9 years $\pm 8.5 \mathrm{SD}$ for controls; $\mathrm{p}=0.70 ; \mathrm{SE}=$ 2.13 Student's t test) and gender (20 men $(80.0 \%)$ for cases vs. 36 men $(74.0 \%)$ for controls; $p=$ 0.45; Chi-square test) is noted. Insall-Salvati ratio concerning patellar height was different between cases and controls $(1.29 \pm 0.23$ vs. $1.13 \pm 0.20 ; p$ 
$=0.002)$, so does Caton-Deschamps index $(1.3 \pm$ 0.20 vs. $1.1 \pm 0.30 ; p=0.004)$. Patellar morphology represented by subchondral Wiberg angle, was greater in patients with proximal tendinitis vs. controls $(139.8 \pm 6.5$ vs. $134.5 \pm 7.1$; $\mathrm{p}=0.003$ ). Femorotibial angle (valgus) was greater in patients with proximal tendinitis vs. controls $\left(5.4^{\circ} \pm 2.6\right.$ vs. $\left.3.1^{\circ} \pm 3.3: \mathrm{p}=0.003\right)$. Rest of all MRI parameters showed no significant differences between cases and controls. Logistic regression analysis is done between MRI parameters of cases and controls with $p$ value < 1.0 for assessing association with patellar tendinitis. Three parameters were found to be associated with patellar tendinitis. They include the Insall-Salvati ratio (odds ratio (OR) of 1.3 (95\% CI 1.0, 1.7); $\mathrm{p}=0.04)$, the femorotibial angle $(\mathrm{OR}=1.2(95 \% \mathrm{CI} 1.1,1.5) ; \mathrm{p}=0.01)$, and the subchondral Wiberg angle $(\mathrm{OR}=1.1(95 \% \mathrm{CI}$ $1.0,1.1) ; \mathrm{p}=0.02)$.

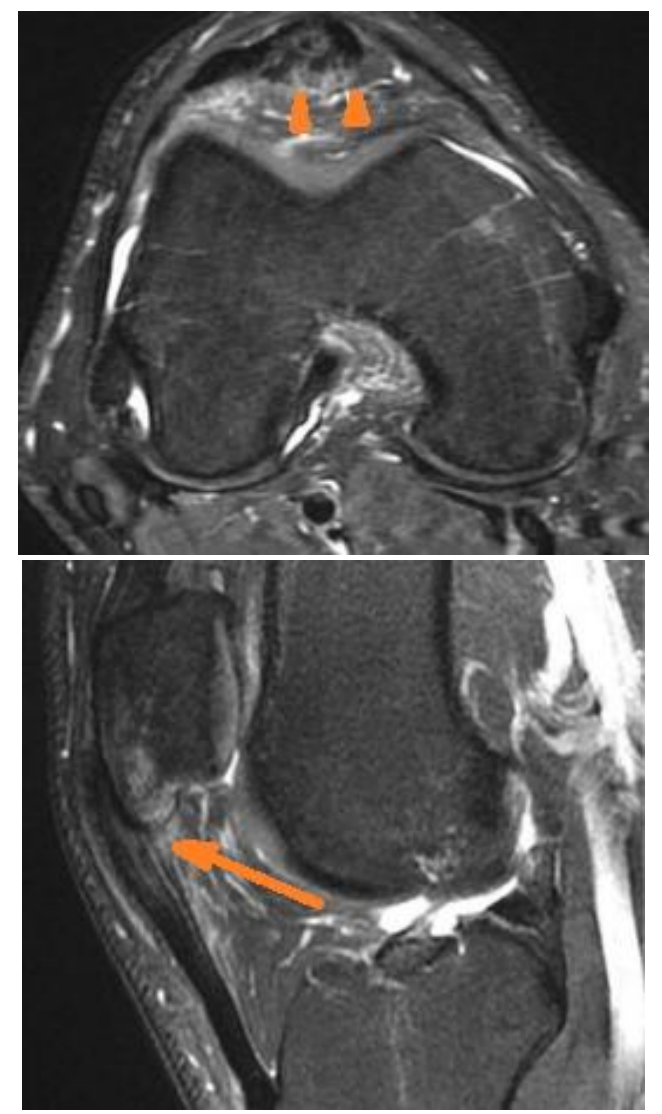

Figure 1: A case of Proximal Patellar Tendinitis. Axial and Sagittal MR images show intrasubstance signal changes of deep portions of the proximalpatellar tendon, thickening of the proximal aspect of the patellar tendon.

Table 1: MRI parameters for Patellofemoral Anatomy \& alignment

\begin{tabular}{|c|c|c|c|c|}
\hline MRI Parameter & Cases (Mean \pm SD) & Controls (Mean \pm SD) & Total (Mean \pm SD) & $\mathrm{P}$ value \\
\hline TTTG $(\mathrm{mm})$ & $11.4 \pm 3.5$ & $10.98 \pm 2.3$ & $11.19 \pm 2.9$ & 0.81 \\
\hline Patellar Tilt $\left(^{\circ}\right)$ & $11.13 \pm 5.8$ & $8.96 \pm 4.57$ & $15.61 \pm 5.2$ & 0.08 \\
\hline Lateral Patellar Displacement (mm) & $3.9 \pm 3.0$ & $3.7 \pm 2.2$ & $3.8 \pm 2.6$ & 0.52 \\
\hline Femorotibial angle $\left(^{\circ}\right)$ & $5.4 \pm 2.6$ & $3.1 \pm 3.3$ & $4.25 \pm 2.9$ & 0.003 \\
\hline Caton-Deschamps index & $1.3 \pm 0.2$ & $1.1 \pm 0.3$ & $1.2 \pm 0.25$ & 0.004 \\
\hline Insall-Salvati ratio & $1.29 \pm 0.23$ & $1.13 \pm 0.2$ & $1.21 \pm 0.215$ & 0.002 \\
\hline Subchondral Wiberg angle $\left({ }^{\circ}\right)$ & $139.8 \pm 6.5$ & $134.5 \pm 7.1$ & $137.15 \pm 6.8$ & 0.003 \\
\hline Subchondral Wiberg Index & $0.56 \pm 0.05$ & $0.55 \pm 0.03$ & $0.555 \pm 0.04$ & 0.28 \\
\hline Medial/lateral trochlea length ratio & $1.42 \pm 0.32$ & $1.41 \pm 0.29$ & $1.41 \pm 0.31$ & 0.89 \\
\hline Lateral trochlea inclination angle $\left({ }^{\circ}\right)$ & $26.4 \pm 3.3$ & $25.7 \pm 4.1$ & $26.05 \pm 3.7$ & 0.46 \\
\hline Trochlear sulcus angle $\left(^{\circ}\right)$ & $131.2 \pm 5.4$ & $132.5 \pm 6.7$ & $131.85 \pm 6.1$ & 0.40 \\
\hline
\end{tabular}

\section{Discussion}

In our retrospective study, we observed that some MRI Knee parameters related to patellar height (Insall-Salvati ratio \& Caton-Deschamps index) and anatomy (subchondral Wiberg angle) \& femorotibial alignment (femorotibial angle), showed statistically significant differences between proximal patellar tendinitis patients and controls. These parameters were also associated with proximal patellar tendinitis.

Risk factors leading to proximal patellar tendinitis include both extrinsic and intrinsic of which extrinsic plays a major role. Extrinsic factors include Training errors (fatigue, distance, hill work, intensity, \& technique), Surfaces, Environmental, Footwear and equipment ${ }^{16}$. Many 
intrinsic factors can potentially alter the load applied on extensor mechanism of the knee including Patella \& patellar tendon. This relationship would need a study.

A previous case control study evaluated students with risk factors of patellar tendinitis which used leg alignment parameters including the Q-angle, leg-length discrepancy, and the medial tibial intercondylar distance. This study is done using clinical evaluation but not with imaging ${ }^{17}$. Martens et al. study noted that soccer \& volleyball were the sports in which two-thirds of all their patients where patellar tendinopathy were involved $^{18}$.

In a recent study on basketball and volleyball athletes found that athletes displaying iliotibial band and shank-forefoot alignment above the normal cut-off values had more chance of patellar tendon abnormalities noted on ultrasound, but no significant association is reported for knee alignment parameters ${ }^{19}$. Culvenor et al found that infrapatellar fat pad size is associated with patellar tendinopathy but not patellar alignment parameters which includes trochlear sulcus angle, Insall-Salvati ratio, lateral patellar tilt angle \& lateral patellar displacement ${ }^{20}$. This study was done with the help of MRI imaging. MRI for patellofemoral instability is observed to be a valuable diagnostic tool for assessing anatomy and alignment ${ }^{21}$.

Of all the Patellofemoral alignment parameters we assessed only Insall-Salvati and CatonDeschamps ratios for patellar height have been found to be associated with proximal patellar tendinopathy. Though the InsallSalvati ratio is usually obtained from lateral knee radiographs, MRI measurement have been found to have high reproducibility when done for patellar height. Patellar and Patellar tendon maltracking finally results in tendinitis and we hypothesize that Patella alta which alters the transmitted load to patellar tendon is the reason behind this. However this study could not differentiate whether altered patellar tendon or patellar bone length is responsible for this phenomenon. Altered load to patellar tendon can also occur due to flattened and unstable patella which is evident while assessing subchondral wiberg angle in our study. We recommend more studies in the future for assessing the role of patellar height and anatomy in causing ultimately proximal patellar tendinitis.

Our study also found out femorotibial angles show significant differences between patients with tendinitis and controls. This angle is noted to be greater in patellar tendinitis patients. So, this valgus malalignment results in lateral patellar dislocating force \& alter the load on extensor mechanism leading to tendinitis. Since MRI was done in supine position, this study may not be a good test femorotibial alignment. There is also considerable overlap between cases and controls in measuring this angle. So, femorotibial angle cannot be used to distinguish tendinitis patients from controls.

One limitation of the study is due to its retrospective nature. As we cannot control for the specific type of physical activity or sport like jumping, running and kicking being played by subjects. Since symptomatic controls are included, they might alter their physical activity to decrease load on knees and potentially avoid patellar tendinitis. Next we ignored presence or absence of joint effusion as exclusion criteria which could impact some of the patellofemoral alignment parameters. Lastly, there is not enough power to assess TT-TG distance relationship with patellar tendinitis as regular knee protocol cannot include entire axial stack.

\section{Conclusion}

Wiberg angle and patellar height (Insall-Salvati ratio and Caton-Deschamps index) were greater in patients with proximal patellar tendinitis with significant association. Further studies are needed to confirm the relationships and help in better management of patients, especially in athletes to formulate preventive strategies. 


\section{References}

1. Khan KM, Cook JL, Taunton JE, Bonar F. (2000) Overuse tendinosis, not tendinitis. Part 1: new paradigm for a difficult clinical problem. Phys Sportsmed. 28:3848.

2. Orava S, Leppilahti J. (1999) Overuse injuries of tendons inathletes. In: Jakob RP, Fulford P, Horan F, eds. EuropeanInstructional Course Lectures. London: The British Editorial Society of Bone and Joint Surgery.

3. Orava S. (1980) Exertion injuries due to sports and physical exercise: A clinical study of nontraumatic overuse injuries of the musculoskeletal system of athletes and keepfit athletes. MD Thesis, Oulu University, Finland

4. Martens M,Wouters P, Burssens A, Mulier JC. (1982) Patellar tendinitis: Pathology and results of treatment. ActaOrtop Scand. 53:445-450.

5. Ferretti A, Ippolito E, Mariani P, Puddu G. Jumper's knee. Am JSports Med. 1983;11:58-62.

6. Stanish WD, Rubinovich RM, Curwin S. Eccentric exercise inchronic tendinitis. Clin Orthop Relat Res 1986:65-8.

7. Cook JL, Khan KM, Harcourt PR, Grant M, Young DA,Bonar SF. (1997) A cross sectional study of 100 athletes withjumper's knee managed conservatively and surgically. Br JSports Med. 31:332-336.

8. Kujala U. (1986) Knee exertion injuries in adolescents andyoung athletes. Publication of the Social Insurance Institution of Finland ML

9. Makhsous M, Lin F, Koh JL, Nuber GW, Zhang LQ. In vivo andnoninvasive load sharing among the vasti in patellar malalignment.Med Sci Sports Exerc. 2004;36:1768-75.

10. Charles MD, Haloman S, Chen L, Ward SR, Fithian D, Afra R.Magnetic resonance imaging-based topographical differences between control and recurrent patellofemoral instability patients. AmJ Sports Med. 2013;41:374-84.

11. Khan KM, Bonar F, Desmond PM, Cook JL, Young DA, Visentini PJ, Fehrmann MW, Kiss ZS, O'Brien PA, Harcourt PR, Dowling RJ, O'Sullivan RM, Crichton KJ, Tress BM, Wark JD. (1996) Patellar tendinosis (jumper's knee): findings at histopathologic examination, US, and MR imaging. Victorian Institute of Sport Tendon Study Group. Radiology. 200:821-827.

12. Fredberg U, Bolvig L. (1999) Jumper's knee-review ofliterature. Scand J Med Sci Sports. 9(2):66-73.

13. Almekinders LC, Vellema JH, Weinhold PS. (2002) Strain patterns in the patellar tendon and the implicationsfor patellar tendinopathy. Knee Surg Sports Traumatol Arthrosc. 10(1):2-5

14. Fredberg U, Bolvig L. (1999) Jumper's knee-review ofliterature. Scand J Med Sci Sports. 9(2):66-73

15. Pope CF. (1992) Radiologic evaluation of tendon injuries. Clin Sports Med. 11:579599.

16. Renström P, Johnson RJ. (1985) Overuse injuries in sports:a review. Sports Med. 2:316-333

17. Witvrouw E, Bellemans J, Lysens R, Danneels L, Cambier D.Intrinsic risk factors for the development of patellar tendinitis inan athletic population. A twoyear prospective study. Am J SportsMed. 2001;29:190-5.

18. Martens M, Wouters P, Burssens A, Mulier JC. (1982) Patellar tendinitis: Pathology and results of treatment. Acta Ortop Scand. 53:445-450.

19. Mendonca LD, Verhagen E, Bittencourt NF, Goncalves GG, Ocarino JM, Fonseca ST. Factors associated with the presence of 
patellar tendonabnormalities in male athletes. J Sci Med Sport.2016;19:389-94.

20. Culvenor AG, Cook JL, Warden SJ, Crossley KM. Infrapatellar fatpad size, but not patellar alignment, is associated with patellar tendinopathy. Scand J Med Sci Sports. 2011;21:e405-11.

21. Charles MD, Haloman S, Chen L, Ward SR, Fithian D, Afra R. Magnetic resonance imaging-based topographical differences between control and recurrent patellofemoral instability patients. Am J Sports Med. 2013;41:374-84. 